\title{
CARACTERIZACIÓN Y EVALUACIÓN DE LA SUSTENTABILIDAD DE FINCAS EN ALTO URUBAMBA, CUSCO, PERÚ*
}

\section{CHARACTERIZATION AND EVALUATION OF FARM SUSTAINABILITY IN ALTO URUBAMBA, CUSCO, PERU}

\author{
Isaías Merma ${ }^{1}$ y Alberto Julca ${ }^{2}$
}

\begin{abstract}
Resumen
El estudio se llevó a cabo en la provincia de La Convención, Cusco - Perú, en la región geográfica de selva alta conocida como Alto Urubamba. Tiene como objetivo evaluar las características prediales y medir la sustentabilidad de fincas a través de indicadores adaptados al lugar. Se recogió información de campo tanto en términos biofísicos y socio-económicos y se analizó en variables seleccionadas, a partir de una base de datos aplicando programas como SPSS, MINITAB y Análisis Cluster. Los resultados muestran que el área promedio de las fincas es de 12.38 ha, la producción en la región es diversificada con un patrón de cultivos prevalentes como el café, cacao, té, coca y frutales tropicales para la venta, junto a cultivos anuales y crianzas para el autoconsumo. La economía de los agricultores es crítica, el ingreso económico promedio en la zona está por debajo de los índices oficiales. Hay problemas ecológicos como quemas e incendios forestales (21.7\%), deforestación (15.1\%) y sequías prolongadas (15.1\%). La presión de uso sobre la tierra es moderada, los sistemas productivos de subsistencia representan el $46.2 \%$ y los semi intensivos para la venta 53.8 \%. La evaluación de sustentabilidad califica como sustentables a las fincas con cultivos de mango (2.87), cacao (2.82), plátano (2.80), cítricos (2.63), papaya (2.57) y como no sustentables a las fincas con té y coca, por no haber alcanzado valores mínimos (2).

Palabras clave: caracterización de fincas, selva alta, cultivos prevalentes, evaluación de sustentabilidad, sistemas de cultivo, sistemas de producción.
\end{abstract}

\begin{abstract}
The study was conducted in the province of La Convencion, Cusco - Peru, in a geographical region of the high jungle known as Alto Urubamba. This research aims to evaluate the farm characteristics and measure their sustainability through local adapted indicators. Field data were collected both in biophysical and socio-economical terms and analyzed in selected variables from a database using programs such as SPSS, MINITAB and Cluster Analysis. The results show that the average farm area is 12.38 ha and that there is a diversified production in the region with a pattern of prevalent crops such as coffee, cocoa, tea, coca and tropical fruits that are destined for sale, accompanied by annual crops and livestock for subsistence. The economy of the farmers is critical; the average income in the area is below the official numbers. There are ecological problems such as wildfire (21.7\%), deforestation (15.1\%) and prolonged droughts (15.1\%). The land-use pressure is moderate, subsistence production systems represent $46.2 \%$, while semiintensive production systems destined for sale represent 53.8\%. According to the sustainability evaluation, sustainable farms are those that produce mango (2.87), cocoa (2.82), banana (2.80), citrus (2.63) and papaya (2.57), and unsustainable farms are those that produce coca and tea which do not reach minimum values (2).
\end{abstract}

Key words: farms characterization, high forest, prevalent crops, sustainability evaluation, cropping systems, production systems.

\footnotetext{
* Sub proyecto de la investigación: "Evaluación y diseño de fincas en selva alta bajo sistemas de cultivos prevalentes en La Convención - Cusco” tesis doctoral en Agricultura Sustentable. Escuela de Post Grado de la Universidad Nacional Agraria La Molina - Perú.
} 


\section{Introducción.}

Una carencia frecuente en los planes y proyectos de desarrollo rural es el conocimiento de los sistemas agrícolas de una región (Apollin \& Eberhart, 1999). Muchas tecnologías no son adoptadas por los productores porque no se adecúan a sus problemas más sentidos ni a sus objetivos, por lo que antes de generar y difundir alternativas tecnológicas es necesario evaluar los sistemas productivos (Andreoli \& Tellarini, 2000).

Los estudios sobre evaluación de fincas y agroecosistemas tropicales en selva alta en suelos con limitaciones de ladera y sequía estacional son escasos, encontrándose pocas experiencias en la literatura científica (Mendoza \& Prabhu, 2000).

El estudio de las fincas y sus agroecosistemas, permitirá comprender los efectos de la intervención del hombre sobre el ecosistema (Altieri et al., 2011), explicará las estrategias campesinas de sobrevivencia frente a la crisis de la agricultura (López et ál., 2007) y facilitará la propuesta de nuevas alternativas de desarrollo.

Las metodologías de "investigación de sistemas prediales o de finca” enfatizan la comprensión de los sistemas agrícolas tradicionales, realizan exámenes en el terreno que incluyen entrevistas a los agricultores respecto a las características del predio y analizan los métodos de producción (Altieri, 1997). Es necesario reconocer las tecnologías utilizadas, las cuales en su mayoría están adaptadas a las condiciones del lugar (León-Velarde \& Quiroz, 1994).

La caracterización consiste en la descripción del predio de acuerdo a las dimensiones de análisis agroecológico, técnico-productivo y socio-económico (Apollin y Eberhart, 1999). Implica determinar un conjunto de variables que distinguen a una finca o unidad de producción que la hace diferente a otras, por lo tanto se considera como una etapa determinante en la investigación de sistemas agrarios (León-Velarde \& Quiroz, 1994; FAO / USAC, 1995).

El Análisis de los Sistemas Agrarios con base en la sustentabilidad se centra especialmente a nivel de finca (Lovell et al., 2002, Pacini et al., 2002), por que en este ámbito es donde se expresan más nítidamente las limitantes a la producción agrícola, lo que permite abordar y discutir acerca de las barreras técnicas, ecológicas y sociales más relevantes (Guzmán \& Alonso, 2007).

La sustentabilidad se define como la habilidad de un sistema de mantener la productividad aun cuando sea sometido a "stresess o perturbaciones" (Conway, 1994). Según Dixon \& Fallow (1989) sustentabilidad (o, más correctamente, cosecha sostenida) significa utilizar el recurso sin reducir su stock físico.

La evaluación de la sustentabilidad es afectada por la propia multidimensión del concepto (ecológica, económica, socio cultural), por lo tanto requiere un abordaje holístico (Andreoli \& Tellarini, 2000) y sistémico, donde predomine el análisis multicriterio (Mendoza \& Prabhu, 2000; Evia \& Sarandón, 2002).

Un indicador es una variable, una medida, un valor, un índice (Gallopin, 1997) que califica la sostenibilidad. La interpretación y validación de los indicadores dependerá de cómo ellos armonizan los requerimientos del medio ambiente "biofísico" con los de "economía” y “sociedad” (Rigby et al., 2001). Para Pannell \& Schilizzi (1999) los indicadores de "criterio múltiple” son más recomendables para las fincas en el ámbito rural.

Los investigadores que se han ocupado en proponer y aplicar indicadores de sustentabilidad coinciden en que no existen indicadores universales que puedan ser utilizados para cualquier situación (Costanza \& Patten, 1995; Hardy, 1997). Por lo tanto, estos deben ser adecuados a los objetivos propuestos, los indicadores deben ser simples, sintéticos y robustos, adaptados a los agroecosistemas de la región (Girardin et al, 1999).

El café, cacao y los frutales tropicales fueron instalados en los mejores suelos de la provincia de La Convención-Cusco, mostrando mayor eficiencia en el uso de los recursos en el caso de los dos primeros y mejores índices de rentabilidad en el caso de los frutales (IMA-Región Cusco, 2005). En cambio los cultivos de té y coca ocupan suelos con limitaciones de ladera y sequía estacional (Merma, 1997).

Las preguntas de investigación son: ¿Cuál es la racionalidad socio-económica y agro-técnica que caracteriza a los sistemas agrícolas de la región del Alto Urubamba? y ¿Son sustentables las fincas con cultivos prevalentes?

Se plantea la hipótesis: "las fincas con cultivos prevalentes de café, cacao y frutales tropicales son más sustentables que las fincas que tienen té y coca”. Los objetivos del presente estudio fueron: evaluar las características de los predios agrícolas en el Alto Urubamba, La Convención-Cusco y medir la sustentabilidad de fincas con cultivos prevalentes.

\section{Materiales y métodos.}

El estudio se llevó a cabo en la zona denominada "Alto Urubamba", que comprende los distritos de Santa Ana, Maranura y parte de Huayopata, Santa Teresa y Echarate de la provincia La ConvenciónCusco.

Es una región geográfica localizada en la parte sur oriental del Perú, entre los paralelos $11^{\circ} 15^{\prime}$ y $13^{\circ} 30^{\prime}$ Latitud Sur y los meridianos $72^{\circ}$ y $74^{\circ}$ Longitud Oeste. El ámbito territorial es de $6525.38 \mathrm{Km}^{2}$ comprende la cuenca alta del río Urubamba que recorre la provincia de sur a norte hacia la vertiente atlántica, formando parte del sistema hidrográfico del río Amazonas.

La región se encuentra en un rango altitudinal entre 600 a 2200 msnm; con una temperatura media anual de $25.4{ }^{\circ} \mathrm{C}$; la precipitación anual entre 989 y 
1200 mm, con una época lluviosa de octubre a marzo y una seca de abril a septiembre. La fisiografía dominante (selva alta) puede definirse como vertiente escarpada, ondulada y accidentada con laderas entre 15 y $70 \%$ de gradiente. cultivo) en los cuales se midió la sustentabilidad aplicando indicadores "tipo multicriterio". La metodología incluye los siguientes pasos:

a) Construcción de indicadores.- Los indicadores se construyeron de acuerdo a la metodología y el

Tabla 1. Indicadores y sub indicadores para cada dimensión de sustentabilidad empleando la metodología “tipo multicriterio” (Sarandón, 2002).

\begin{tabular}{lll}
\hline DIMENSIÓN & DIMENSIÓN & DIMENSIÓN SOCIO - \\
ECONÓMICA (IK) & ECOLÓGICA (IE) & CULTURAL (ISC) \\
\hline
\end{tabular}

\section{A.- autosuficiencia alimentaria:}

A1.-diversificación de la producción

A2.- superficie de producción para el autoconsumo

\section{B.- ingreso económico neto mensual por familia. \\ C.- riesgo económico: \\ C1.- diversificación para la venta \\ C2.- número vías de comercialización \\ C3.- dependencia de insumos externos}

\section{A.- conservación de la vida del suelo:}

A1.- restos orgánicos en el suelo

A2.- tiempo de cobertura del suelo con vegetación

A3.- diversificación de cultivos

\section{B.- riesgo de erosión: \\ B1.- pendiente predominante \\ B2.- cobertura vegetal \\ B3.- orientación de los surcos}

C.- manejo de la biodiversidad:

C1.- biodiversidad espacial

C2.- biodiversidad temporal

\section{A.- satisfacción de las necesidades básicas}

A1.- acceso a la salud y cobertura sanitaria

A2.- acceso a la educación

A3.- vivienda

A4.- servicios

\section{B.- aceptabilidad del} sistema de producción.

\section{C.- integración social a sistemas organizativos. \\ D.- conocimiento y conciencia ecológica.}

\section{Caracterización de los sistemas prediales.}

Consistió en la descripción y análisis de variables seleccionadas inherentes a la producción agropecuaria de la zona. Para esto, se recogió información directa de los productores lo cual sirvió para caracterizar los sistemas agrícolas a nivel de finca describiendo los aspectos técnicos, ecológicos y socioeconómicos más relevantes.

Se consideró a 106 agricultores como la población muestral, estratificados según cinco distritos, a los cuales se aplicaron encuestas a base de cuestionarios previamente elaborados; también se trabajó con grupos focales con el fin de obtener información primaria.

La información se ordenó y se analizó en sus tres dimensiones: biofísico, económico y social. El análisis comprende la elaboración de una base de datos a partir de las encuestas y la aplicación de paquetes estadísticos como SPSS V. 15, MINITAB V. 14, y para encontrar los usos de la tierra se efectuó Análisis Cluster.

Identificación de cultivos prevalentes.

La caracterización permitió identificar los cultivos prevalentes de la región tomado en cuenta el área, la producción, importancia económica y la preferencia de los agricultores.

Medición de la sustentabilidad de fincas.

Habiéndose identificado ocho cultivos prevalentes, se seleccionaron 24 fincas representativas (tres por marco conceptual propuesto por Sarandón (2002), siguiendo los lineamientos de Smyth \& Dumansky (1995) y Astier et al., (2002). Se eligieron indicadores y sub indicadores para cada dimensión considerada, que fueran fáciles de obtener, de interpretar y que brindaran la información necesaria (Tabla 1).

b) Estandarización y ponderación de los indicadores.- Para permitir la comparación de las fincas y facilitar el análisis, los datos fueron estandarizados mediante su transformación a una escala para cada indicador de 0 a 4 , siendo 4 el mayor valor de sustentabilidad y 0 el más bajo (Sarandón et al., 2006).

Posteriormente los indicadores fueron ponderados multiplicando el valor de la escala por un coeficiente de acuerdo a la importancia relativa o peso de cada variable respecto a la sustentabilidad.

c) Descripción y ponderación de los indicadores elegidos.- En base al análisis de las condiciones locales y al grado de conocimiento del investigador sobre la zona, se hizo algunas modificaciones a la metodología planteada por Sarandón et al. (2006) referidos a la descripción de sub indicadores y sus unidades de medida y al peso de los indicadores, lo que se refleja en las fórmulas finales.

Los datos parcelarios y de finca se obtuvieron mediante observaciones de campo y entrevistas. Se presentan las fórmulas para cada dimensión analizada. 


$$
\begin{aligned}
& \text { Indicador Económico (IK) }=\frac{2((\mathrm{~A} 1+\mathrm{A} 2) / 2)+2 \mathrm{~B}+(\mathrm{C} 1+\mathrm{C} 2+\mathrm{C} 3) / 3}{5} \\
& \text { Indicador Ecológico (IE) } \quad=\frac{(2 \mathrm{~A} 1+\mathrm{A} 2+\mathrm{A} 3) / 4+(2 \mathrm{~B} 1+\mathrm{B} 2+\mathrm{B} 3) / 4+(\mathrm{C} 1+\mathrm{C} 2) / 2}{3} \\
& \text { Indicador Sociocultural (ISC) }=\frac{2((2 \mathrm{~A} 1+2 \mathrm{~A} 2+\mathrm{A} 3+2 \mathrm{~A} 4) / 7)+2 \mathrm{~B}+\mathrm{C}+\mathrm{D}}{6}
\end{aligned}
$$

El valor de cada macro indicador es un cociente cuyo numerador es la sumatoria ponderada de indicadores y sub indicadores considerados y el denominador es el número de variables tomando en cuenta su ponderación.

Por ejemplo, para el macro indicador económico (IK), se ha ponderado con 2 los indicadores: autosuficiencia alimentaria (A) e ingreso económico neto mensual por familia (B), porque califican directamente la condición económica familiar y el indicador riesgo económico (C) con ponderación simple. A todo el numerador se divide por cinco, que es el número de casos ponderados $(2 \mathrm{~A}+2 \mathrm{~B}+\mathrm{C})$. De manera similar se procede con las dimensiones ecológica y sociocultural.

Finalmente, con los datos de los macro indicadores, se calcula el índice de sustentabilidad general, valorando a las tres dimensiones por igual: Índice de Sustentabilidad General $($ ISGen $)=(\mathrm{IK}+\mathrm{IE}$ + ISC) / 3

Condiciones.

a) Para considerar a una finca como sustentable el Índice de Sustentabilidad General (ISGen) debe ser mayor a 2 y además;

b) Ninguna de las tres dimensiones evaluadas debe tener un valor menor a 2 (Sarandón et al., 2006).

\section{Resultados y discusión.}

Cultivos prevalentes.

Considerando el área y su aporte en la economía de la zona, se identificaron los cultivos prevalentes: café, cacao, coca, té y frutales tropicales como plátano, cítricos, papaya y mango (Tabla 2). Otras

\begin{tabular}{|c|c|c|c|c|c|c|c|c|c|}
\hline \multicolumn{2}{|c|}{ Cultivos } & Distritos & Huayopata & Maranura & $\begin{array}{l}\text { Santa } \\
\text { Teresa }\end{array}$ & $\begin{array}{l}\text { Santa } \\
\text { Ana }\end{array}$ & Echarate & $\begin{array}{l}\text { Promedio } \\
\text { por finca }\end{array}$ & $\begin{array}{l}\text { Promedio } \\
\text { nacional }\end{array}$ \\
\hline \multirow{2}{*}{\multicolumn{2}{|c|}{ Café }} & Extensión (ha) & 0,95 & 2,63 & 2,80 & 2,54 & 3,63 & 2,51 & \\
\hline & & Rdto. (qq/ha) & 12,40 & 15,33 & 14,41 & 13,22 & 13,57 & 13,79 & 16,45 \\
\hline \multirow{2}{*}{\multicolumn{2}{|c|}{ Cacao }} & Extensión (ha) & 0,00 & 0,35 & 0,00 & 0,20 & 2,84 & 1,13 & \\
\hline & & Rdto. (qq/ha) & 0,00 & 10,37 & 0,00 & 9,12 & 8,54 & 9,34 & 13,13 \\
\hline \multirow{2}{*}{\multicolumn{2}{|c|}{ Coca }} & Extensión (ha) & 0,24 & 0,67 & 0,59 & 0,24 & 0,23 & 0,40 & \\
\hline & & Rdto. (@/ha) & 41,09 & 56,47 & 48,57 & 42,06 & 54,29 & 48,50 & 75,00 \\
\hline \multirow{2}{*}{\multicolumn{2}{|c|}{ Té }} & Extensión (ha) & 1,68 & 0,00 & 0,00 & 0,00 & 0,00 & 1,68 & \\
\hline & & Rdto. (kg. HF) & 2444,26 & 0,00 & 0,00 & 0,00 & 0,00 & 2444,26 & 3000 \\
\hline \multirow{2}{*}{\multicolumn{2}{|c|}{ Achiote }} & Extensión (ha) & 0,33 & 0,65 & 0,33 & 0,40 & 0,93 & 0,53 & \\
\hline & & Rdto. (qq/ha) & 7,67 & 11,83 & 6,94 & 12,61 & 9,97 & 9,80 & 8,70 \\
\hline \multirow{8}{*}{ Frutales } & \multirow{2}{*}{ Plátano } & Extensión (ha) & 0,20 & 0,21 & 0,37 & 0,31 & 1,20 & 0,46 & \\
\hline & & Rdto. (t/ha) & 6,30 & 6,87 & 5,98 & 8,20 & 9,45 & 7,36 & 12,85 \\
\hline & \multirow{2}{*}{ Cítricos } & Extensión (ha) & 0,24 & 1,03 & 0,24 & 0,32 & 1,68 & $\mathbf{0 , 7 0}$ & \\
\hline & & Rdto. (t/ha) & 11,86 & 9,24 & 8,71 & 11,60 & 13,75 & 11,03 & 14,93 \\
\hline & \multirow{2}{*}{ Papaya } & Extensión (ha) & 0,00 & 0,20 & 0,00 & 0,37 & 0,92 & 0,50 & \\
\hline & & Rdto. (t/ha) & 0,00 & 14,13 & 0,00 & 10,98 & 17,99 & 14,37 & 14,40 \\
\hline & \multirow{2}{*}{ Mango } & Extensión (ha) & 0,00 & 0,51 & 0,15 & 0,13 & 0,35 & 0,29 & \\
\hline & & Rdto. (t/ha) & 0,00 & 27,36 & 5,00 & 7,08 & 6,37 & 11,45 & 18,00 \\
\hline
\end{tabular}

Tabla 2. Extensión y rendimiento de cultivos prevalentes en el Alto Urubamba, La Convención - Cusco (n=106, año 2008).

Fuente: Elaborado en base a la encuesta para agricultores, 2008.

Leyenda: qq=quintales; @= arrobas; HF = hoja fresca. 
actividades de menor importancia económica son los cultivos anuales (yuca, maíz, taro, frijol), ganadería extensiva y crianza de aves y cuyes.

En el Alto Urubamba la extensión promedio de los cafetales es de 2,51 ha por finca con un rendimiento de $13,79 \mathrm{qq} / \mathrm{ha}$, muy similar a lo encontrado por Rosado (2005) de 4,20 ha y 13,90 qq/ha para cafetales orgánicos de la misma zona.

Los rendimientos de los cultivos son bajos en comparación a los promedios nacionales. Así, en el caso del café $(13,79 \mathrm{qq} / \mathrm{ha})$ es menor al promedio nacional (16,45 qq/ha); en cacao (9,34 qq/ha) es inferior a 13,13 qq/ha; la productividad del té $(2.444,26 \mathrm{~kg}$ hoja fresca/ha) es menor al promedio nacional (3.000 kg hoja fresca/ha). Similar comportamiento tienen los frutales.

La baja productividad de los cultivos se debe al manejo poco tecnificado y escasa inversión en la finca. Al productor le interesa combinar varios cultivos en la finca, de acuerdo a una estrategia de diversificación para disminuir impactos económicos o biológicos que harían peligrar el ingreso económico de su familia.

Hay una vinculación de los distritos con la calidad de los cultivos: Huayopata con la producción del té (se denomina la capital tealera del Perú), Maranura con la producción de mango y coca, Echarate con el cacao (un tipo de cacao nativo llamado “chuncho”). El café se cultiva en todos los distritos, resaltando los sitios mayores a 1200 msnm que producen cafés de excelente calidad (“café de altura”).

Uso de la tierra.

La extensión total de las fincas varía según los distritos: Huayopata (7,14 ha); Santa Ana (9,90 ha); Santa Teresa (10,03 ha); Maranura (11,99 ha) y Echarate (22,88 ha). El promedio para la región es de 12,38 ha. La tierra se usa en forma extensiva, cada finca es un mosaico de cultivos, pastos, bosques $\mathrm{y}$ eriazos.

La presión de uso sobre la tierra es moderada, el café es el principal cultivo de la región. Los bosques naturales (2,58 ha por finca) son la reserva familiar para la provisión de leña y madera. Los bosques secundarios (purmas) prosperan por el clima tropical que favorece la vegetación espontánea; a esto se añade el uso de árboles de sombra como: Pacae mono (Inga adenophylla), Albizia (Albizzia sumatrana), Pisonay (Erithryna falcata) y Chamba (Leucaena trichodes).

Muchos suelos calificados como áreas de protección (IMA-Región Cusco, 2005) están siendo utilizados para cultivar café (23\%), coca (17 \%), maíz (15 \%) y achiote (13 \%). Como consecuencia, estos territorios se encuentran en conflicto de uso. A esto se suma la deforestación en la zona, acelerando problemas de erosión de suelos y pérdida de biodiversidad.

Según el análisis Cluster (Figura 1), existen tres grupos de uso de la tierra relacionados según su extensión. El grupo (1) constituido por café y bosques, los mismos que presentan mayor extensión; el grupo (2) por frutales, cacao, cultivos anuales y pastos; el grupo (3) por achiote, coca, té y eriazos con menor extensión.

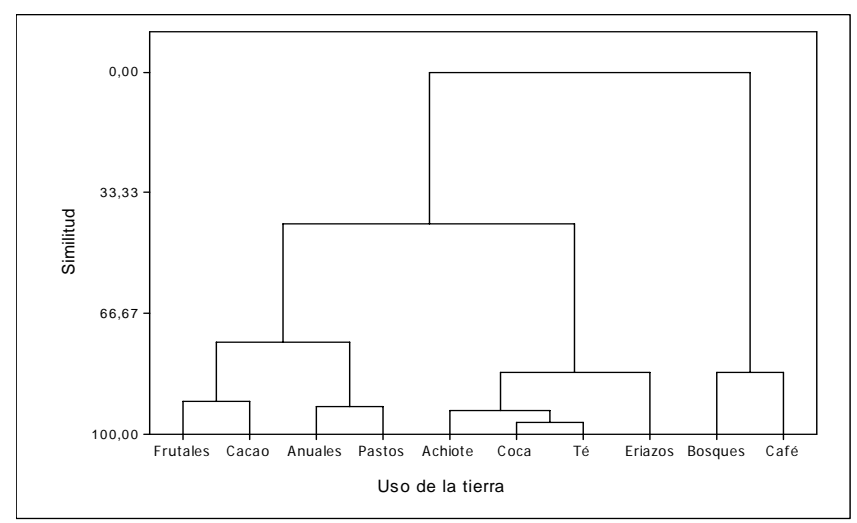

Figura 1. Análisis Cluster del uso de la tierra en fincas del Alto Urubamba, La Convención - Cusco ( $\mathrm{n}=106$, año 2008).

\section{Destino de la producción.}

El destino de la producción agropecuaria se muestra en la Figura 2. Los cultivos permanentes se destinan más para la venta (15\% al autoconsumo); los cultivos anuales en cambio, se consumen más en la propia zona (80 \% de autoconsumo); la misma tendencia tienen las crianzas (75\% destinados al autoconsumo).

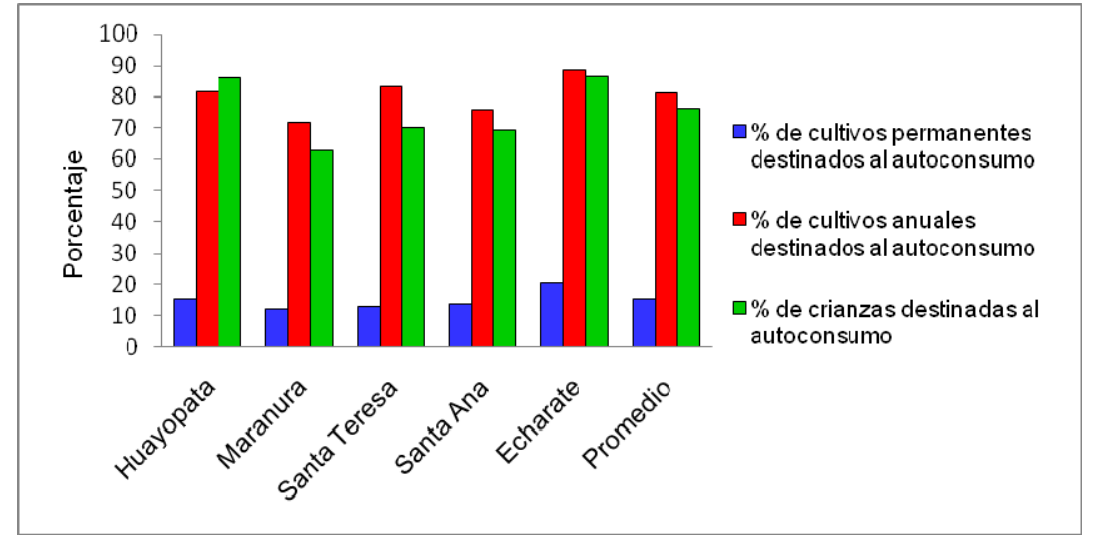

Figura 2. Porcentaje de cultivos y crianzas destinados para el autoconsumo en el Alto Urubamba, La Convención - Cusco (n=106, 
Análisis económico.

La economía de los agricultores en el Alto Urubamba es crítica; los ingresos por familia y por persona son bajos en todos los distritos (Tabla 3). El ingreso mensual percápita promedio de la zona ( $\mathrm{S} /$. 197,71 nuevos soles) está por debajo del sueldo mínimo vital (S/. 550,00 nuevos soles), índice oficial que fijó el Estado Peruano para el año 2010. El ingreso diario de \$2,25 por persona, es considerado bajo (Banco Mundial, 2008). Se verifica así, un estado de pobreza en la zona rural del Alto Urubamba.

Según el gráfico de cajas (Figura 3), Huayopata, Maranura y Santa Ana tienen medianas similares respecto a la utilidad neta anual. Santa Teresa y Echarate muestran mejores ingresos por familia. Existen algunos agricultores atípicos que tienen ingresos muy altos para la zona, en Maranura un productor cafetalero (20) y un productor de mango (19); en Santa Ana, uno con producción

Tabla 3. Análisis económico de ingreso neto por finca en el Alto Urubamba, La Convención - Cusco (n=106, año 2008).

\begin{tabular}{|c|c|c|c|c|c|}
\hline Distrito & $\begin{array}{c}\text { Total } \\
\text { Utilidad } \\
\text { Neta Anual } \\
\text { Familiar } \\
\text { (S/.) }\end{array}$ & $\begin{array}{c}\text { Total } \\
\text { Utilidad } \\
\text { Neta } \\
\text { Mensual } \\
\text { Familiar } \\
\text { (S/.) }\end{array}$ & $\begin{array}{c}\mathrm{N}^{\circ} \\
\text { personas } \\
\text { que } \\
\text { dependen } \\
\text { de la } \\
\text { finca }\end{array}$ & $\begin{array}{c}\text { Ingreso } \\
\text { mensual } \\
\text { percápita } \\
(\mathrm{S} / .)\end{array}$ & $\begin{array}{c}\text { Ingreso } \\
\text { mensual } \\
\text { percápita } \\
(\$)^{*}\end{array}$ \\
\hline Huayopata & $5.696,94$ & 474,75 & 3,59 & 132,24 & 45,60 \\
\hline Maranura & $8.150,60$ & 679,22 & 4,87 & 139,47 & 48,09 \\
\hline Santa Ana & $8.981,75$ & 748,48 & 3,80 & 196,97 & 67,92 \\
\hline Sta. Teresa & $8.342,08$ & 695,17 & 3,29 & 211,30 & 72,86 \\
\hline Echarate & $14.013,67$ & $1.167,81$ & 3,67 & 318,20 & 109,73 \\
\hline Promedio & $9.037,01$ & 753,08 & 3,84 & 197,71 & 68,18 \\
\hline
\end{tabular}

Fuente: Elaborado en base a la encuesta para agricultores, 2008.

* Tipo de cambio: \$ 1,00 US = 2,90 nuevos soles. cacaotalero (64); estos son agricultores con fincas grandes (> 20 ha) con capital disponible y tecnologías eficientes como riego tecnificado, abonamiento, suficiente mano de obra e infraestructura productiva.

Arreglos espaciales y cronológicos de cultivos.

Las figuras 4 y 5 muestran los distintos arreglos de cultivos. En cuanto a los arreglos espaciales, los monocultivos (23,6 \%) son menos preferidos que el conjunto de policultivos $(76,4 \%)$. Estos últimos se presentan en diversos arreglos como: cultivos asociados (32,1 \%), cultivos intercalados $(13,2 \%)$, multiestrato desordenado (12,3\%), cultivos de borde $(11,3 \%)$ y cultivos en franjas $(8,5 \%)$. diversificada (59) y en Echarate un productor

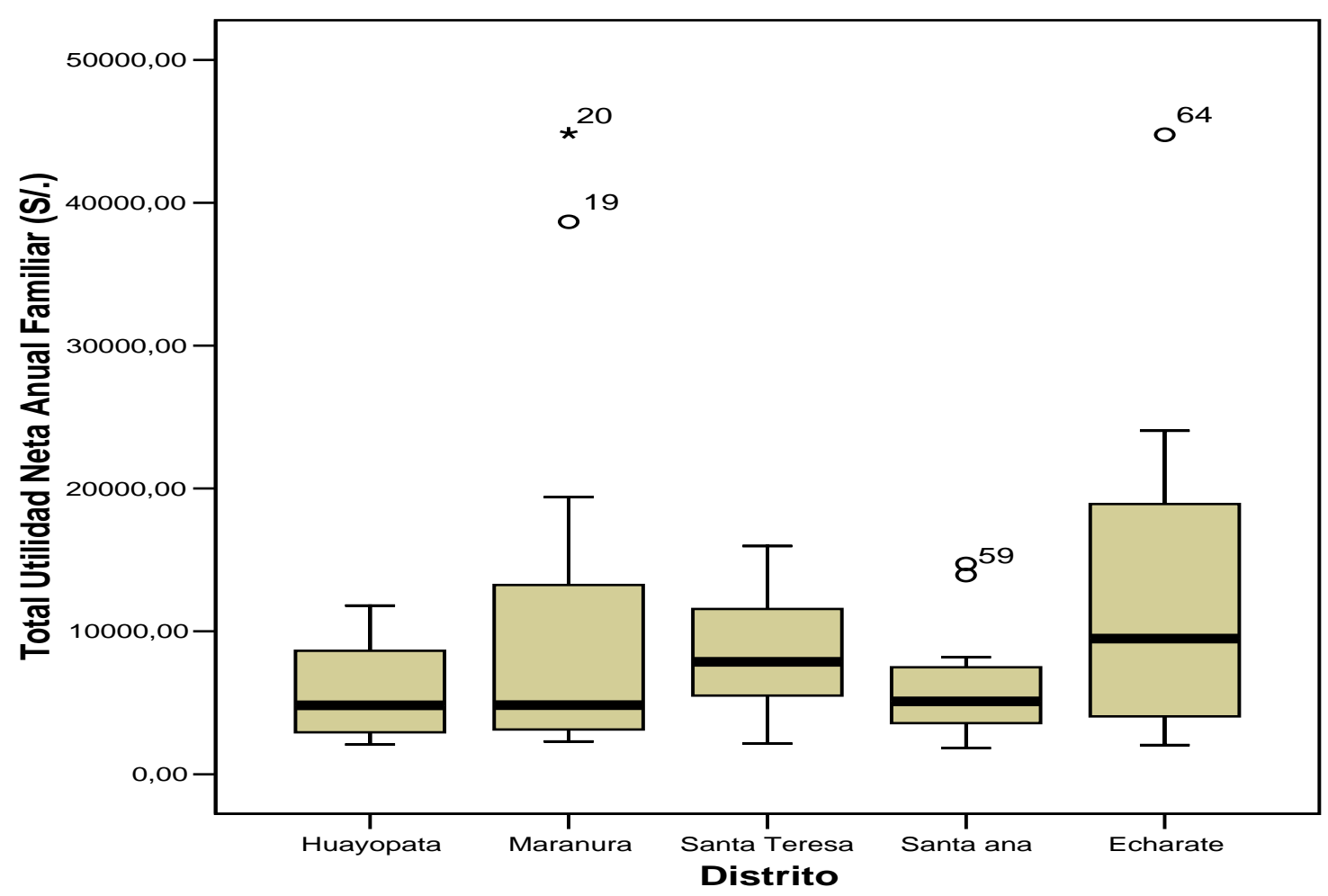

Figura 3. Ingreso neto anual familiar según distritos en el Alto Urubamba, La Convención - Cusco ( $\mathrm{n=106}$, año 2008). 


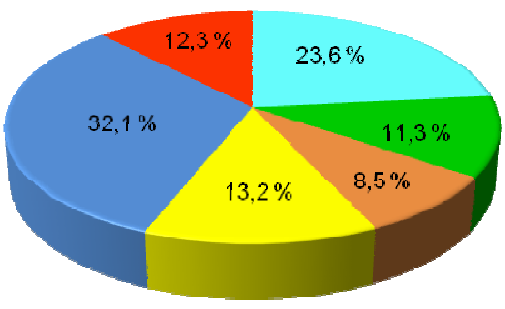

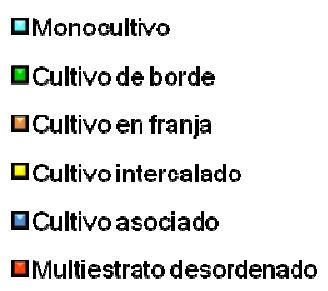

Figura 4. Arreglos espaciales de cultivos en fincas del Alto Urubamba, La Convención - Cusco ( $\mathrm{n}=106$, año 2008).

La preferencia por los policultivos en la zona tiene varias razones: 1. Es una estrategia de diversificación de productos en el campo; 2. Los principales cultivos son de hábito perenne que requieren de árboles de sombra 3. La vegetación natural del ecosistema tropical se regenera y mantiene una foresta boscosa.

Es conocido que los policultivos conducidos por pequeños productores obtienen mayor productividad en términos de productos aprovechables por unidad de área que los monocultivos con el mismo nivel de gestión. Según Dorward (1999) las ventajas de rendimiento pueden variar desde 20 a $60 \%$.

Los arreglos cronológicos que practican los agricultores son: siembra libre con barbecho (47,2 \%), siembra y cosecha escalonadas (16,0\%), cosecha escalonada (13,2 \%), relevo (11,3\%), siembra escalonada (5,7 \%), siembra estrecha sin barbecho (5,7 \%) y simultánea (0,9 \%). Las lluvias definen los arreglos, sobre todo en los cultivos de ciclo corto (anuales). Una vez cosechado se deja el terreno en barbecho o descanso, así los sistemas se adaptan al calendario agrícola anual.

Sistemas de cultivo.

Existe una gran diversidad de sistemas de cultivo en las fincas, debido a la "estrategia multicultivo" que practican los agricultores en forma tradicional y al clima favorable de la zona para acoger muchos cultivos y crianzas. Los sistemas de cultivo predominantes son: café con árboles de sombra (18,9 $\%)$, café asociado con plátano (11,3\%), coca sin sombra (7,6 \%), crianza de animales menores (6,6 \%), maíz intercalado con yuca (5,7\%), café asociado con

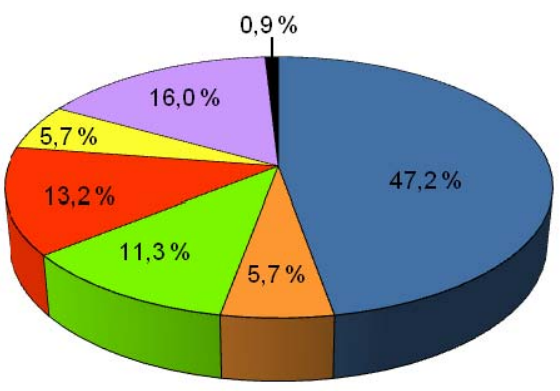

$$
\begin{aligned}
& \square \text { Libre con barbecho } \\
& \square \text { Estrecho sin barbecho } \\
& \square \text { En relevo } \\
& \square \text { Cosecha escalonada } \\
& \square \text { Siembra escalonada } \\
& \square \text { Siembra y cosecha } \\
& \text { escalonada }
\end{aligned}
$$

Figura 5. Arreglos cronológicos de cultivos en fincas del Alto Urubamba, La Convención - Cusco (n=106, año 2008). cacao (3,8 \%), café con cítricos (3,8 $\%)$, cacao con plátano (3,8 \%) entre otros (Figura 6).

La presencia de estos sistemas ratifica la predominancia de los policultivos sobre los monocultivos y de los cultivos sobre las crianzas. La vegetación promueve la formación de multiestratos por la presencia de árboles de sombra y la asociación de cultivos. Estas son características innatas de la biodiversidad que mantiene un ecosistema tropical.

$\mathrm{Al}$ productor le interesa combinar varios cultivos en la finca, de acuerdo a una estrategia de versificación para disminuir impactos económicos o necesida. El incremento de los rendimientos es una el crecimiento, superar la pobreza y mejorar la seguridad alimentaria (Banco Mundial, 2008).

Stemas de producción.

La Figura 7 muestra una síntesis de los sistemas de en el Alto Urubamba. Los sistemas de subsistencia representan el 46,2 \% y

Los productores adoptan uno o varios sistemas según el capital existente, el nivel tecnológico y el grado de inserción en el mercado. Según Malagón \& raguer (2001) "hay fincas que como sistemas están a están por lograrlo; en ambos casos las . existen formas agrarias empresariales y formas campesinas con prácticas de producción no tensificadas.

Se verifica así la dualidad entre la producción de cultivos perennes para la venta y cultivos transitorios autoconsumo. Durante los últimos años la captando mayor interés hacia una agricultura comercial. De esta manera, se han incrementado las áreas de café, cacao, papaya, plátano y cítricos (IMARegión Cusco, 2005) reemplazando los sistemas de subsistencia y de recolección que aún existen en la región.

Problemas ecológicos.

La figura 8 muestra los principales problemas ecológicos en el Alto Urubamba. Las causas más frecuentes son de origen antrópico. Según Merma (1997) "la región ha soportado una colonización descontrolada sin planificación ni apoyo del Estado; 
el migrante serrano se ha asentado en un ecosistema tropical diferente a su lugar de origen y como resultado es un manejo inexperto de los recursos naturales”. A esto se añade la condición de ecosistema frágil de la zona debido a la fisiografía accidentada, a una geodinámica activa y a un régimen de lluvias muy concentrado en ciertas épocas del año y períodos de sequías prolongados.

Si bien la agricultura y la colonización desordenada en la zona han contribuido a acelerar los problemas ecológicos, la respuesta no consiste en desacelerar el crecimiento agrícola. Muchas innovaciones tecnológicas e institucionales pueden dar lugar a una agricultura más sostenible (Banco Mundial, 2008). En la zona se puede promover el riego tecnificado, la agricultura orgánica, tecnologías agroforestales, gestión empresarial, entre otros.

Armonizar producción y medio ambiente es el gran reto que enfrenta actualmente la humanidad, una alternativa es desarrollar sistemas sostenibles de producción a nivel de finca con bajos insumos externos, bajos costos y gastos de operación (Campos et al., 2005).

Sustentabilidad de fincas con cultivos prevalentes.

Los resultados (Tabla 4), muestran que el índice de sustentabilidad general (IS-g) es mayor a 2 (umbral mínimo) en todas las fincas estudiadas; pero el cultivo de coca no alcanza el valor mínimo en la dimensión ecológica $(1,50)$ y el té en las dimensiones económica $(1,84)$ y sociocultural $(1,90)$, por lo que no son consideradas sustentables. Las fincas con café $(2,50)$, cacao $(2,82)$ y los frutales como plátano $(2,80)$, cítricos (2,63), papaya $(2,57)$ y mango $(2,87)$ superaron el valor mínimo por lo que son consideradas sustentables.

Los frutales tropicales como el mango, plátano, cítricos y papaya muestran buenos valores de sustentabilidad debido al valor económico en los mercados locales y regionales y en el caso del mango además por su valoración ecológica.

El café muestra bajos índices, debido a la variabilidad del precio del producto cosechado y se mantiene sustentable por su raigambre social, el agricultor está identificado con este cultivo. El cacao obtiene buenos valores debido a que es un cultivo tradicional en la zona, mantiene precios estables, es un árbol perenne que protege el suelo y promueve la biodiversidad.

Mención aparte es el caso de la coca, que a pesar de no alcanzar sustentabilidad general, obtiene valores aceptables en la dimensión económica y socio cultural, debido a que es un cultivo ancestral en la zona. La hoja cosechada por los agricultores es adquirida por el Estado Peruano para fines de consumo tradicional.

La validez de la aplicación de indicadores utilizando el análisis multicriterio puede ser discutida para el caso del Alto Urubamba; lo importante es cumplir ciertas condiciones tal como Masera et al. (1999) señalan: la evaluación de sustentabilidad se lleva a cabo en: (a) sistemas de manejo específicos en un lugar geográfico y bajo un contexto social; (b) una escala espacial (parcela, unidad de producción o cuenca) previamente determinada; y (c) una escala temporal.

En este estudio se ha cumplido dichas condiciones, así: (a) Los sistemas agrícolas son conducidos por pequeños agricultores que habitan en la selva del Alto

Tabla 4. Evaluación de la sustentabilidad de fincas con cultivos prevalentes utilizando el análisis multicriterio, en Alto Urubamba, La Convención - Cusco (n=3, año 2009).

\begin{tabular}{lllllllllllllllll}
\hline Finca & AA & IM & RE & IK & VS & RE & MB & IE & $\begin{array}{l}\text { SN } \\
(2)\end{array}$ & $\begin{array}{l}\text { Ac } \\
(2)\end{array}$ & IS & CE & ISC & IS-g & $\begin{array}{l}\text { Sustenta- } \\
\text { bilidad }\end{array}$ \\
\hline Café & 2,5 & 2 & 2,9 & $\mathbf{2 , 3 8}$ & 2,3 & 2 & 2,9 & $\mathbf{2 , 4 0}$ & 2,3 & 2,5 & 3,4 & 3,4 & $\mathbf{2 , 7 3}$ & $\mathbf{2 , 5 0}$ & Sí & \\
Cacao & 3 & 3 & 2,6 & $\mathbf{2 , 9 2}$ & 2,9 & 2,7 & 2,9 & $\mathbf{2 , 8 3}$ & 2,5 & 2,8 & 3,1 & 2,5 & $\mathbf{2 , 7 0}$ & $\mathbf{2 , 8 2}$ & Sí & No \\
Coca & 3,5 & 2,5 & 2,2 & $\mathbf{2 , 8 4}$ & 1,6 & 1,1 & 1,8 & $\mathbf{1 , 5 0}$ & 2,9 & 2,9 & 3,5 & 2,9 & $\mathbf{3 , 0 0}$ & $\mathbf{2 , 4 5}$ & No \\
Té & 2,5 & 1 & 2,2 & $\mathbf{1 , 8 4}$ & 2,2 & 2,7 & 2,5 & $\mathbf{2 , 4 7}$ & 1,9 & 1,8 & 1,9 & 2,1 & $\mathbf{1 , 9 0}$ & $\mathbf{2 , 0 7}$ & No & Sí \\
Plátano & 3 & 3 & 2,9 & $\mathbf{2 , 9 8}$ & 2,3 & 2,3 & 3 & $\mathbf{2 , 5 3}$ & 2,7 & 3 & 2,8 & 3,1 & $\mathbf{2 , 8 8}$ & $\mathbf{2 , 8 0}$ & Sí \\
Cítricos & 3 & 3,5 & 2,5 & $\mathbf{3 , 1 0}$ & 2,3 & 2,1 & 2,5 & $\mathbf{2 , 3 0}$ & 2,5 & 2,5 & 2,4 & 2,6 & $\mathbf{2 , 5 0}$ & $\mathbf{2 , 6 3}$ & Sí \\
Papaya & 3 & 3,7 & 3,1 & $\mathbf{3 , 3 0}$ & 2,1 & 1,9 & 2,3 & $\mathbf{2 , 1 0}$ & 2,3 & 2,3 & 2,2 & 2,4 & $\mathbf{2 , 3 0}$ & $\mathbf{2 , 5 7}$ & Sí & \\
Mango & 3 & 3 & 3 & $\mathbf{3 , 0 0}$ & 2,8 & 3,5 & 3 & $\mathbf{3 , 1 0}$ & 2,4 & 2,6 & 2,3 & 2,7 & $\mathbf{2 , 5 0}$ & $\mathbf{2 , 8 7}$ & Sí & \\
\hline
\end{tabular}

Fuente: Sarandón et al. (2006) y evaluaciones de campo.

Nota: Valores promedio $<2$ indican sistemas no sostenibles.

Leyenda:

IK= indicador económico: $\mathrm{AA}=$ autosuficiencia alimentaria; $\mathrm{IM}=$ ingreso económico neto mensual por familia; $\mathrm{RE}=$ riesgo económico.

IE= indicador ecológico: VS= vida del suelo; $\mathrm{RE}=$ riesgo de erosión; $\mathrm{MB}=$ manejo de la biodiversidad.

ISC= indicador sociocultural: $\mathrm{SN}=$ satisfacción de las necesidades básicas; Ac= aceptabilidad del sistema; IS= integración social; $\mathrm{CE}=$ conocimiento y conciencia ecológica.

IS-g= índice de sustentabilidad general. 


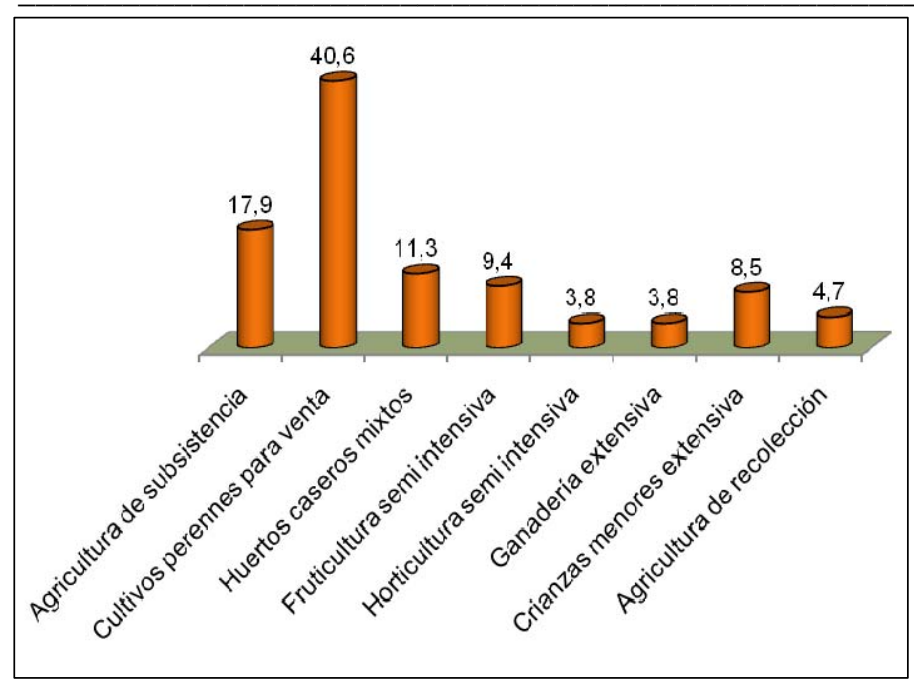

Figura 7. Sistemas de producción prevalecientes (\%) en el Alto Urubamba, La Convención - Cusco ( $n=106$, año 2008).

Urubamba-Cusco; (b) Se ha evaluado agroecosistemas con cultivos prevalentes a nivel de finca; (c) La evaluación fue hecha durante el año 2009.

\section{Conclusiones.}

El Alto Urubamba es una zona de selva alta cuya principal actividad económica es la agricultura. En dicha región se ha evaluado las características prediales de acuerdo a las dimensiones de análisis agro-ecológico, técnico-productivo y socio-económico y se ha evaluado la sustentabilidad de fincas con cultivos prevalentes.

La economía de los agricultores es crítica, la baja rentabilidad de la agricultura viene generando pobreza en las familias campesinas de la zona. Es necesario intensificar la producción de cultivos de óptima calidad intrínseca aprovechando las ventajas comparativas que oferta el medio ambiente. El Estado debe mejorar la infraestructura productiva, brindar soporte financiero y asistencia técnica para reconvertir la agricultura de subsistencia a una comercial con gestión empresarial.

En la zona hay problemas ecológicos debido a la intervención antrópica. La necesidad de obtener ingresos a corto plazo y el uso de tecnologías inapropiadas por los agricultores, viene ocasionando la pérdida de recursos especialmente suelo y vegetación. Es necesario cuantificar los daños producidos en los ecosistemas.

La evaluación de la sustentabilidad de fincas con cultivos prevalentes reporta que las fincas con café, cacao y frutales como plátano, cítricos, papaya y mango superan el valor mínimo (2) por lo que son consideradas sustentables, mientras que las fincas con el cultivo de coca no alcanzan el valor mínimo en la dimensión ecológica (1.50) y con el té en las dimensiones económica (1.84) y sociocultural (1.90) por lo que no son consideradas sustentables para las condiciones en estudio.
Los resultados confirman la hipótesis planteada: "las fincas con cultivos prevalentes de café, cacao y frutales tienen mayores índices de sustentabilidad que las fincas con té y coca”.

Los indicadores de sustentabilidad tipo multicriterio utilizados en este estudio demostraron su validez y adaptación al medio; son simples, confiables y replicables, por lo que se recomienda su uso en condiciones similares.

\section{Agradecimientos.}

A la FEPCACYL, APROCAV, TE HUYRO y cooperativas bases de COCLA; a sus agricultores y técnicos por la valiosa información de campo y apoyo logístico.

\section{Literatura citada.}

Altieri M.A. 1997. Enfoque Agroecológico para el Desarrollo de Sistemas de Producción Sostenibles en los Andes. Ed. CIED. Lima-Perú. 92 p.

Altieri M.A.; Funes M.F.; Petersen P.; Tomic T. \& Medina, Ch. 2011. Sistemas agrícolas ecológicamente eficientes para los pequeños agricultores. Foro Europeo de Desarrollo Rural 2011. Palencia, España. 27 p.

Andreoli M. \& Tellarini V. 2000. Farm sustainability evaluation: methodology and practice. Agriculture, Ecosystems and Environment 77:43-52.

Apollin F. \& Eberhart C. 1999. Análisis y diagnóstico de sistemas de producción en el medio rural. Guía metodológica. CAMAREN, Quito-Ecuador. 239 p.

Astier M., López Ridaura S., Pérez Agis E. \& Masera O.R. 2002. El Marco de Evaluación de Sistemas de Manejo incorporando Indicadores de Sustentabilidad (MESMIS) y su aplicación en un sistema agrícola campesino en la región Purhepecha, México. En Agroecología: El camino hacia una agricultura sustentable (Sarandón SJ, ed.) Ediciones Científicas americanas, cap. 21: 415-430.

Banco Mundial. 2008. Informe sobre el desarrollo mundial 2008: Agricultura para el desarrollo. Bogotá, Colombia. Banco Mundial y Mayol Ediciones. S.A.

Campos M.; Machado H.; Matías Y.; González L.; Sánchez S. \& Duquesne P. 2005. Diagnóstico socioeconómico, ambiental e institucional de una entidad productiva mediante metodologías participativas. Matanzas, Cuba. Rev. Pastos y Forrajes, Vol. 28, No. 4. 331-340.

Conway G.R. 1994. Sustainability in agricultural development: Trade-offs between productivity, stability and equitability. Journal For Farming Systems and Research-Extensions 4, núm. 2: 1-14.

Costanza R. \& Patten B. 1995. Defining and predicting sustainability. Ecological Economics 15, 193-196.

Dixon J.A. \& Fallon L.A. 1989. The concept of sustainability: origins, extensions and usefulness for policy. Society and Natural Resources 2: 73-84.

Dorward A. 1999. Farm size and productivity in Malawian smallholder agriculture. Journal of Development Studies, 35: 141 - 161.

Evia G. \& Sarandón S.J. 2002. Aplicación del método multicriterio para valorar la sustentabilidad de diferentes alternativas productivas en los humedales de la laguna 
Merín, Uruguay. En Agroecología: El camino hacia una agricultura sustentable, (Sarandón SJ, ed.) Ediciones Científicas Americanas, Capítulo 22:431-448.

FAO / USAC. 1995. Caracterización del sistema agrario que comprende la zona de retornados "Nueva Esperanza”, Nentón, Huehuetenango. Guatemala. 65 p.

Gallopin G. 1997. Indicators and their use: information for decision making. In: Moldan, B., Billharz, S. (Eds.), Sustainability Indicators. Report on the Project on Indicators of Sustainable Development. Wiley, Chichester.

Girardin P., Bockstaller C. \& Van der Werf H. 1999. Indicators: Tools to evaluate the environmental impacts of farming systems. Journal of Sustainable Agriculture, Vol. 13 (4) 5-14.

Guzmán C.GI. \& Alonso M.AM. 2007. La investigación participativa en agroecología: una herramienta para el desarrollo sustentable. Asociación Española de Ecología Terrestre, AEET. Santa Fe, Granada. Rev. Ecosistemas 16 (1).

Hardy P. 1997. Measurements and indicators program of the International Institute for Sustainable Development. In: Moldan, B. \& Billharz S. (Eds.). Report of the Project on Indicators of Sustainable Development. Scientific Committe on Problems of the environment (SCOPE) West Sussex. P. 28-32.

IMA-Región Cusco. 2005. Esquema de ordenamiento y demarcación territorial La Convención. Instituto de Manejo del Agua - Región Cusco. Informe GTCIMPLC. 238 p.

León-Velarde C \& Quiroz RG. 1994. Análisis de sistemas agropecuarios; uso de métodos bio-matemáticos. CIRNMA- CONDESAN. Puno, Perú. 238 p.

López F.; Gómez R.; Harvey C.; López M. \& Sinclair F. 2007. Toma de decisiones de productores ganaderos sobre el manejo de los árboles en potreros de Matiguás, Nicaragua. Agroforestería en las Américas $N^{\circ}$ 45: 93100.

Lovell C., Mandondo A. \& Moriarty P. 2002. The question of scale in integrated natural resource management. Conservation Ecology. 5:25.

Malagón R.M. \& Prager M.M. 2001. El enfoque de sistemas: Una opción para el análisis de las unidades de producción agrícola. Universidad Nacional Colombia. Palmira. 190 p.

Masera O., Astier M. \& López-Ridaura S. 1999. Sustentabilidad y manejo de recursos naturales. El Marco de Evaluación MESMIS. GIRA.A.C. México. $109 \mathrm{p}$.

Mendoza G. \& Prabhu R. 2000. Multiple criteria decision making approaches to assessing forest sustainability using criteria and indicators: a case study. Forest Ecology and Management 131:107-126.

Merma M. I. 1997. Identificación de sistemas de producción agrícola en Huayopata, La Convención-Cusco. Tesis M.Sc. Producción Agrícola UNALM.EPG. Lima-Perú. $136 \mathrm{p}$.

Pacini C., Wossink A., Giesen G., Vazzana C. \& Huine R. 2002. Evaluation of sustainability of organic, integrated and conventional farming systems: a farm and fieldscale analysis. Agriculture Ecosystems and Environment. 95: 273.

Pannell, J.D. \& Schilizzi, S. 1999. Sustainable agriculture: A matter of ecology, equity, economic efficiency or expedience? Journal of Sustainable Agriculture, Vol. 13(4).

Rigby, D.; Woodhouse, P.; Young, T. \& Burton, M. 2001. Constructing a farm level indicator of sustainable agricultural practice. Journal Ecological Economics 39, 463-478.

Rosado S.L. 2005. Caracterización de la producción de café orgánico en Perú. Junta Nacional del café. Lima-Perú. $210 \mathrm{p}$.

Sarandón S.J. 2002. El desarrollo y uso de indicadores para evaluar la sustentabilidad de los agroecosistemas. En Agroecología: El camino hacia una agricultura sustentable (Sarandón SJ, ed.) Ediciones Científicas Americanas, capítulo 20: 393-414.

Sarandón S.J., Zuluaga M.S., Cieza R., Gómez C., Janjetic L. \& Negrete E. 2006. Evaluación de la sustentabilidad de sistemas agrícolas de fincas en Misiones, Argentina, mediante el uso de indicadores. Revista Agroecología, Vol. 1: 19-28. España.

Smyth A.J. \& Dumannsky J. 1995. A framework for evaluating sustainable land management. Canadian Journal of Soil Science 75: 401-406. 


\section{ANEXOS: figuras citadas en el texto.}

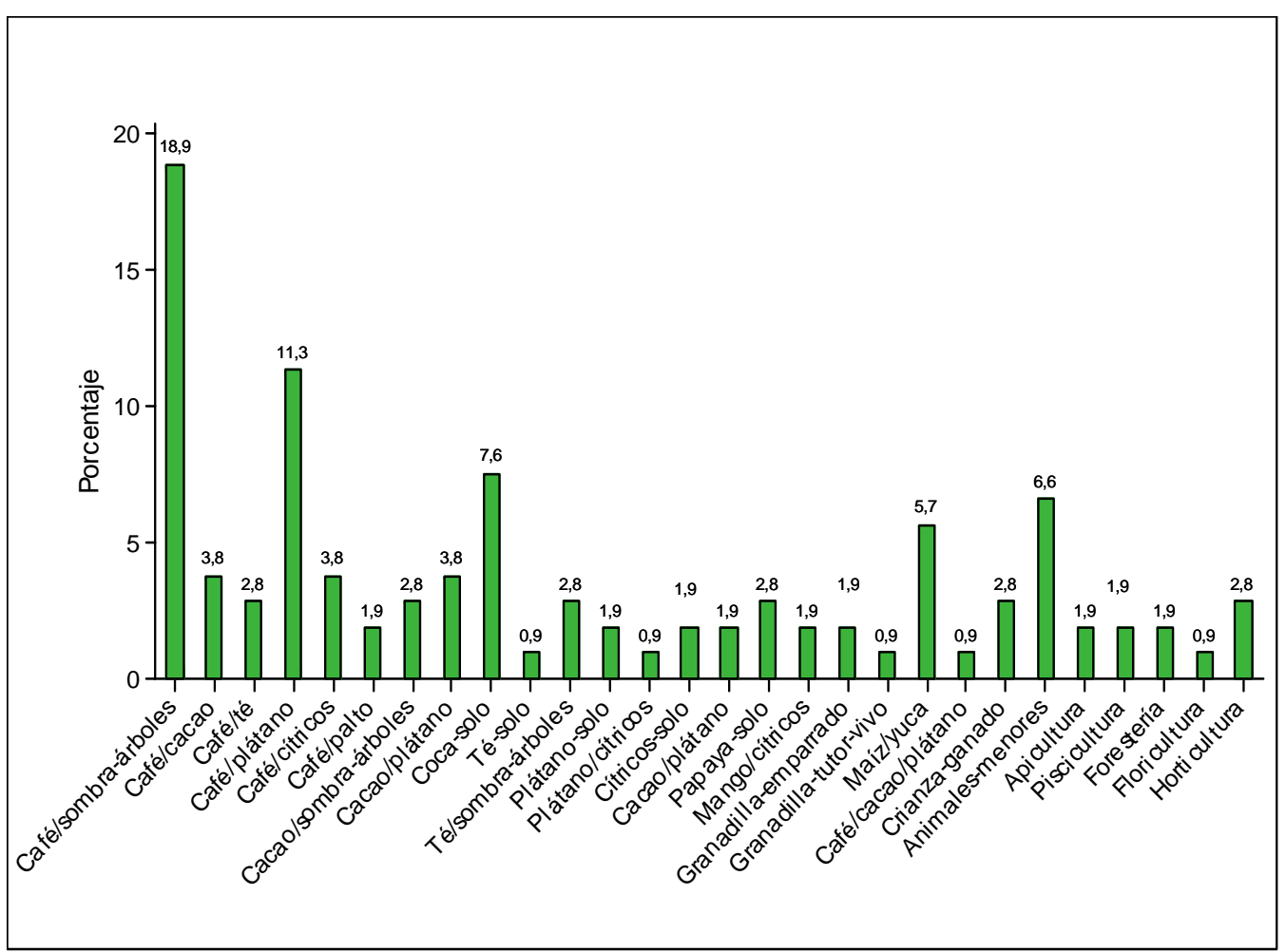

Figura 6. Sistemas de cultivos y crianzas encontrados en fincas del Alto Urubamba, La Convención - Cusco (n=106, año 2008)

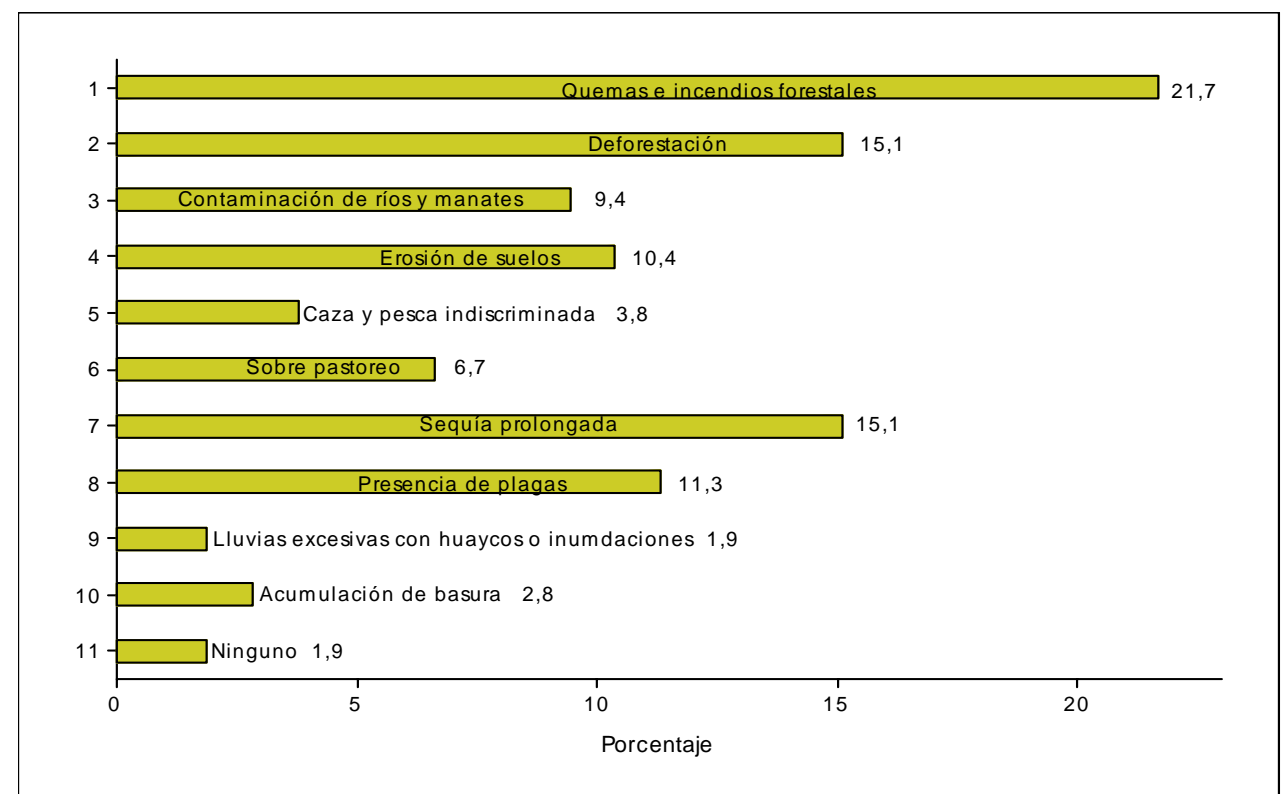

Figura 8. Principales problemas ecológicos encontrados en el Alto Urubamba, La Convención - Cusco (n=106, año 2008).

\footnotetext{
${ }^{1}$ Universidad Nacional San Antonio Abad del Cusco. Facultad de Ciencias Agrarias Tropicales-Quillabamba. Email: i_merma@hotmail.com.

${ }^{2}$ Universidad Nacional Agraria La Molina. Facultad de Agronomía. Departamento Académico de Fitotecnia. Email: ajo@lamolina.edu.pe.
} 